\section{Case Reports in Ophthalmology}

Case Rep Ophthalmol 2018;9:221-226

DOI: 10.1159/000487704

Published online: March 22, 2018
(C) 2018 The Author(s)

Published by S. Karger AG, Basel www.karger.com/cop

This article is licensed under the Creative Commons Attribution-NonCommercial 4.0 International License (CC BY-NC) (http://www.karger.com/Services/OpenAccessLicense). Usage and distribution for commercial purposes requires written permission.

\title{
Intraoperative Adjunctive Mitomycin C and Cryotherapy for Recurrent Conjunctival Papillary Sebaceous Carcinoma
}

\author{
Natsuki Monai ${ }^{\mathrm{a}}$ Reiko Tanabu ${ }^{\mathrm{a}}$ Takayuki Gonome ${ }^{\mathrm{a}}$ Katsunori Yokoi ${ }^{\mathrm{b}}$ \\ Satoshi Urushidate ${ }^{b}$ Satoko Morohashic ${ }^{c}$ Hideaki Hirai $^{d}$ Akira Kurose ${ }^{d}$ \\ Mitsuru Nakazawa ${ }^{a}$ \\ aDepartment of Ophthalmology, Hirosaki University Graduate School of Medicine, \\ Hirosaki, Japan; 'bepartment of Plastic and Reconstructive Surgery, Hirosaki University \\ Graduate School of Medicine, Hirosaki, Japan; 'Department of Pathology and Bioscience, \\ Hirosaki University Graduate School of Medicine, Hirosaki, Japan; dDepartment of \\ Anatomic Pathology, Hirosaki University Graduate School of Medicine, Hirosaki, Japan
}

\section{Keywords}

Sebaceous carcinoma $\cdot$ Mitomycin C $\cdot$ Cryotherapy $\cdot$ Conjunctival sebaceous carcinoma

\begin{abstract}
Purpose: To report a case of recurrent conjunctival papillary sebaceous carcinoma that was successfully treated by a combination of surgical resection, intraoperative topical mitomycin $C$ application, and cryotherapy. Observations: A woman in her 80s developed a yellowish papillary tumor pedunculated from the surface of the upper palpebral tarsal conjunctiva in her left eye. She was histopathologically diagnosed as having sebaceous carcinoma by an excisional biopsy. We performed en bloc resection of the lateral one-third of the posterior lamella including the cutaneous margin of the upper eyelid as well as reconstruction of the defected portion by a switch-flap from the ipsilateral lower eyelid. Histopathologically, because the tumor was restricted to the epithelial region with minimal invasion into the tarsus, we diagnosed the patient to have conjunctival papillary sebaceous carcinoma. Nine months after the surgery, the tumor recurred and was resected and treated by intraoperative mito-
\end{abstract}


mycin C. Four months later, the tumor regrew at the resected margins and was treated by resection combined with mitomycin $C$ and cryotherapy. After these combination treatments, the tumor did not recur for at least 1 year postoperatively. Conclusion and Importance: Although sebaceous carcinoma usually originates from the meibomian gland cells or less frequently from the Zeis or Moll gland cells, it rarely occurs from bulbar or palpebral conjunctival cells. Because sebaceous carcinoma sometimes shows a pagetoid growth pattern, it can recur even after en bloc resection with a negative study for tumor cells at the surgical margins. The recurrent sebaceous carcinoma cells showed an intraepithelial growth pattern. Considering this superficial growth property, it may be effective to apply intraoperative mitomycin $\mathrm{C}$ and cryotherapy treatment combined with surgical resection to reduce the possibility of recurrence of presumed conjunctival papillary sebaceous carcinoma, although mitomycin $\mathrm{C}$ alone seems to be insufficient as an adjunctive treatment.

(C) 2018 The Author(s)

Published by S. Karger AG, Basel

\section{Introduction}

Sebaceous carcinoma usually occurs from meibomian gland cells or, less frequently, from the glands of Zeis or Moll in the eyelid. However, rarely, it can arise from the bulbar or palpebral conjunctiva $[1,2]$. In addition, sebaceous carcinoma sometimes exhibits intraepithelial pagetoid spread. Given this property, sebaceous carcinoma can recur even after "complete resection" with clear margins. Therefore, adjunctive therapies including intraoperative cryotherapy, primary or adjunctive postoperative irradiation, or postoperative topical application of mitomycin $\mathrm{C}$ have been reported [3-5].

We experienced a case of conjunctival papillary sebaceous carcinoma that recurred as a pagetoid spread after surgical excision. Since the patient had had several issues with her general condition, she needed to be treated with as minimally invasive a procedure as possible. Therefore, we performed simple resection of the recurrent tumor combined with intraoperative direct application of mitomycin $\mathrm{C}$ solution. Because the tumor recurred after the treatment, we performed topical mitomycin $\mathrm{C}$ and cryotherapy in addition to simple resection of the tumor. After these treatments, the tumor did not recur for at least 1 year.

To our knowledge, this is the first reported use of simple resection combined with intraoperative application of mitomycin $\mathrm{C}$ and cryotherapy for recurrent conjunctival sebaceous carcinoma.

\section{Case Report}

A woman in her 80s noticed gradually increasing discharge and redness in her left eye associated with slight difficulty in opening the same eye. The discharge disappeared after topical levofloxacin treatment prescribed by an ophthalmologist, who subsequently detected a tumorous mass located behind the upper eyelid. Therefore, she was referred to the Department of Ophthalmology at Hirosaki University Hospital. She had a history of liver cirrhosis caused by hepatitis C, esophageal varix, bronchoectasis, and thrombocytopenia. On examination, a yellowish mass appeared to be located on the palpebral conjunctiva in her left upper eyelid, but neither abnormal findings on the surface of the lid skin nor loss of eyelashes was observed (Fig. 1a). Lid eversion revealed a yellowish multilobulatd papillary mass that was pedunculated from the palpebral tarsal conjunctiva (Fig. 1b). On laboratory examina- 


\section{Case Reports in Ophthalmology}

Case Rep Ophthalmol 2018;9:221-226

DOI: $10.1159 / 000487704$

(c) 2018 The Author(s). Published by S. Karger AG, Basel www.karger.com/cop

Monai et al.: Mitomycin C and Cryotherapy for Recurrent Conjunctival Sebaceous Carcinoma

tion, she had thrombocytopenia $(80,000$ cells/dL). The histopathologic diagnosis of a biopsied specimen was sebaceous carcinoma (Fig. 2a).

Since the tumor appeared to be restricted to the lateral third of the palpebral tarsal conjunctiva without any clinical signs of lymph node metastasis, we performed en bloc resection of the lateral one-third of the posterior lamella of the left upper eyelid, including the marginal cutaneous portion, with a safety margin of $4 \mathrm{~mm}$. All of the frozen sections of the specimens from the resected margins were confirmed to be free of tumor cells. Since other portions of the palpebral conjunctiva did not appear to be inflamed, no map biopsies were carried out. The defected upper eyelid was reconstructed by a switch-flap from the ipsilateral lower eyelid (Fig. 3a). Nine months after the surgery, a Bowen-like abnormal epithelial lesion with a finely vascularized surface was observed on the middle third of the left superior palpebral tarsal conjunctiva (Fig. 3b). The histopathologic diagnosis of a biopsied specimen suggested a recurrence of the sebaceous carcinoma.

Due to the patient's thrombocytopenia, we needed to treat her with as minimally invasive a procedure as possible. Therefore, we performed simple resection of the abnormal epithelial lesion along with the conjunctival surface of the middle third portion of the tarsus. As soon as the tumor was excised, 12 pieces of surgical sponge $(2 \times 2 \times 2 \mathrm{~mm})$ that contained $0.02 \%$ of mitomycin C solution were placed on the surface of the surgically exposed tissue as well as the surrounding intact conjunctival surface for $5 \mathrm{~min}$ in case that there might be residual tumor cells. After the mitomycin $\mathrm{C}$ treatment, all sponges were removed and the tissue was thoroughly rinsed with $200 \mathrm{~mL}$ of sterile saline. The resected surface was left bare. The histopathological findings revealed that the presence of tumor cells at the margin of the resected tissue and the tumor recurred 6 months later. In addition to repeating simple resection and mitomycin $C$ treatment via the same procedure, we performed cryotherapy (three rounds of freezing for about 3-4 s and thawing the surrounding conjunctival tissue). No recurrence occurred after this, and the resected surface was completely covered by the intact conjunctival epithelium (Fig. 3c) for at least 1 year after the third surgery.

A histopathologic examination revealed that the tumor consisted of proliferative atypical vacuolated cells with slight basophilic cytoplasms and atypical nuclei associated with distinct hyperchromatic nucleoli (Fig. 2a). On low magnification, the tumor demonstrated papillary and pedunculated exophytic growth from the palpebral conjunctiva (Fig. 2b). Although a small portion of invasion into the tarsus was found, the tarsal glands were not occupied by carcinoma cells (Fig. 2c).

\section{Discussion}

Sebaceous carcinoma is one of the most common periocular neoplasms in the Asian population. It occurs at rates of about $37.5 \%$ in Japanese [6], 39.0\% in Chinese [7], and $31.2 \%$ in Indians [8] among all periocular tumors, although it accounts for less than 1-5.5\% of the eyelid malignancy in Caucasians $[9,10]$. Because sebaceous gland cells embryologically originate from the conjunctival epithelium, these cells may have a common potential to develop sebaceous carcinoma [2]. To our knowledge, the present case is the second case of presumed conjunctival papillary sebaceous carcinoma. Although the first case demonstrated no recurrence after resection of the posterior lamella of the eyelid [2], our case developed recurrence after the resection of the posterior lamella despite clear surgical margins for the tumor cells. Of note, conjunctival papillary sebaceous carcinoma may also exhibit pagetoid intraepithelial spread, which may lead to recurrence. In addition, because the tumor re- 
Case Reports in
Ophthalmology

Case Rep Ophthalmol 2018;9:221-226

DOI: $10.1159 / 000487704$

(c) 2018 The Author(s). Published by S. Karger AG, Basel www.karger.com/cop

Monai et al.: Mitomycin C and Cryotherapy for Recurrent Conjunctival Sebaceous Carcinoma

curred twice during the clinical course, it is possible that we should have performed map biopsies to yield more precise information regarding the spread of the tumor cells.

The primary treatment of sebaceous carcinoma of the eyelid is surgical excision and subsequent reconstruction when there are no apparent signs of extensive invasion or lymph node metastasis. However, because the tumor cells sometimes exhibit intraepithelial growth or pagetoid spread, the recurrence rate is relatively high, reportedly $32 \%$ even with sufficient safety margins [11]. Therefore, adjunctive therapies, including cryotherapy, irradiation, and chemotherapy using topical instillation of mitomycin $\mathrm{C}$ eye drops are performed if necessary [3]. Although the effect of mitomycin $C$ is controversial, it has been reported that the postoperative use of topical mitomycin $\mathrm{C}$ might be effective in preventing postoperative recurrence $[4,5,12,13]$. However, to our knowledge, there have been no previous reports in which mitomycin C solution was directly applied to the surgical field subsequent to tumor excision.

In contrast, the direct application of mitomycin $\mathrm{C}$ is frequently employed during trabeculectomy procedures to prevent the postoperative proliferation of subconjunctival fibroblasts over the filtration bleb [14-16]. The advantage of the direct application lies in the fact that the surgeon can perform the entire procedures alone, whereas the topical instillation of eye drops requires the patient's cooperation with respect to the frequency and duration of application, which sometimes cannot be maintained due to irritation [4, 13]. Although the intraoperative direct application of mitomycin C may have advantages over topical instillation, its effect was found to be insufficient in our case, given the recurrence of the tumor. Because the addition of cryotherapy successfully prevented the tumor recurrence, a combination of mitomycin $\mathrm{C}$ and cryotherapy may be effective as an adjunctive treatment to prevent recurrence from the putative pagetoid spread of a presumed conjunctival papillary sebaceous carcinoma.

\section{Acknowledgements}

The authors would like to thank Mr. Brian Quinn for his English editing.

\section{Statement of Ethics}

Consent to publish the report was obtained from the patient in writing.

\section{Disclosure Statement}

The following authors have no financial disclosures: N.M., R.T., T.G., K.Y., S.U., S.M., H.H., A.K., M.N.

\section{Funding Sources}

Research funds from Alcon Japan, Santen, K Vision, Novartis, AMO and Pfizer. The present study was supported, in part, by the Grant-in-Aid for Scientific Research (C-16K11313) and the Grant-in-Aid for Young Scientists (B-17K16954) from Japan Society for the Promotion of Science (Tokyo, Japan). 


\section{Case Reports in Ophthalmology}

\section{References}

1 Honavar S, Shields C, Maus M, Shields J, Demirci H, Eagle R Jr: Primary intraepithelial sebaceous gland carcinoma of the palpebral conjunctiva. Arch Ophthalmol 2001;119:764-767.

2 Jacobiec FA, Stagner AM, Nowak MA, Rubin PA: Presumed primary sebaceous carcinoma of the palpebral conjunctiva. JAMA Ophthalmol 2015;113:612-614.

3 Mulay K, Aggaewal E, White VA: Periocular sebaceous gland carcinoma: a comprehensive review. Saudi J Ophthalmol 2013;27:159-165.

4 Shields CL, Naseripour M, Shields J, et al: Topical mitomycin C for pagetoid invasion of the conjunctiva by eyelid sebaceous gland carcinoma. Ophthalmology 2002;109:2129-2133.

5 Tumuluri K, Kourt G, Martin P: Mitomycin C in sebaceous gland carcinoma with pagetoid spread. Br J Ophthalmol 2004;88:718-719.

6 Xu XL, Li B, Sun XL, et al: Eyelid neoplasms in the Beijing Tongren eye centre between 1997 and 2006. Ophthalmic Surg Lasers Imaging 2008;39:367-372.

7 Ohba H, Aoki Y, Kubota S, Kanai N, Tsuru T: Incidence of benign and malignant lesions of eyelid and conjunctival tumors. Nihon Ganka Gakkai Zasshi 2005;109:573-579.

8 Kale SM, Patil SB, Khare N, Math M, Jain A, Jaiswal S: Clinicopathological analysis of eyelid malignancies - a review of 85 cases. Indian J Plast Surg 2012;45:22-28.

9 Cook BE Jr, Bartley GB: Epidemiologic characteristics and clinical course of patients with malignant eyelid tumors in an incidence cohort in Olmsted County. Minnesota Ophthalmol 1999;106:746-750.

10 Kass LG, Hornblass A: Sebaceous carcinoma of the ocular adnexa. Surv Ophthalmol 1989;33:477-490.

11 Ratz JL, Luu-Duong S, Kulwin DR: Sebaceous carcinoma of the eyelid treated with Mohs surgery. J Am Acad Dermatol 1986;14:668-673.

12 Rudkin AK, Muecke JS: Mitomycin-C as adjuvant therapy in the treatment of sebaceous gland carcinoma in high-risk locations. Clin Exp Ophthalmol 2009;37:352-356.

13 Frucht-Pery J, Sugar J, Baum J, et al: Mitomycin C treatment for conjunctival-corneal intraepithelial neoplasia: a multicenter experience. Ophthalmology 1997;104:2085-2093.

14 Kitazawa Y, Kawase K, Matsushita H, Minobe M: Trabeculectomy with mitomycin. A comparative study with fluorouracil. Arch Ophthalmol 1991;109:1693-1698.

15 Palmer SS: Mitomycin as adjunct chemotherapy with trabeculectomy. Ophthalmology 1991;98:317321.

16 Matsuda T, Tanihara H, Hangai M, Chihara E, Honda Y: Surgical results and complications of trabeculectomy with intraoperative application of mitomycin C. Jpn J Ophthalmol 1996;40:526-532.
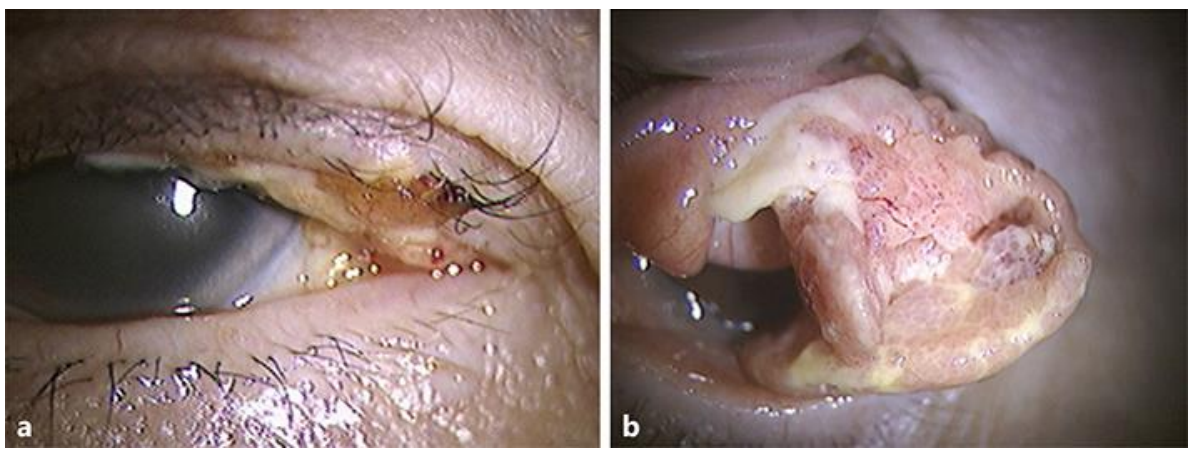

Fig. 1. The edge of a tumorous mass is found at the lower margin of the upper eyelid. There is no loss of eye lashes (a). On lid eversion, there is a yellowish multilobulated papillary tumor behind the lid. The tumor is pedunculated from the tarsal palpebral conjunctiva (b). 


\section{Case Reports in Ophthalmology}

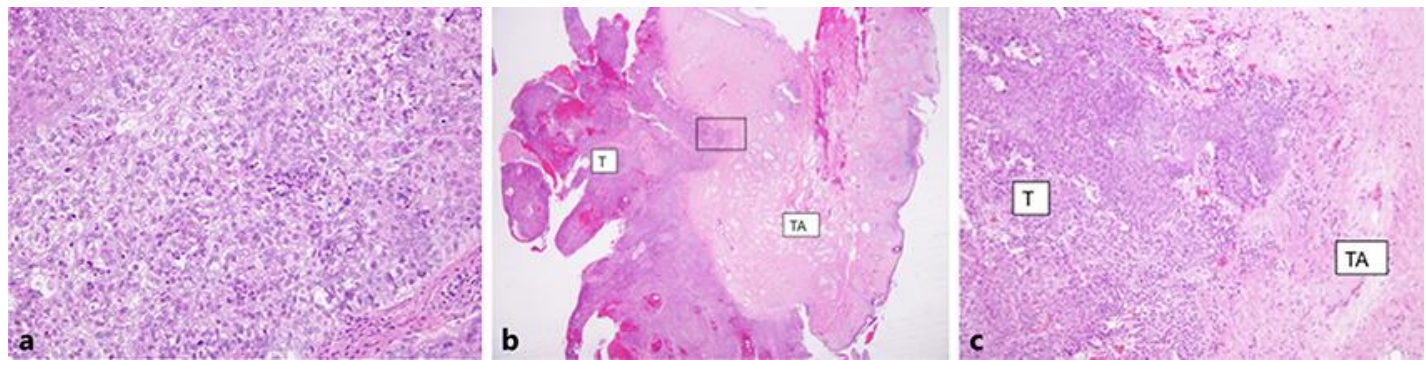

Fig. 2. A histopathologic section (hematoxylin-eosin, original magnification $\times 200$ ) from the biopsied specimen shows that the tumor consists of proliferative atypical vacuolated cells with slight basophilic cytoplasms and atypical nuclei associated with hyperchromatic nucleoli (a). A histopathologic section (hematoxylin-eosin, original magnification $\times 12.5$ ) from the resected specimen shows that the tumor demonstrated papillary and pedunculated exophytic growth from the palpebral conjunctiva (b). Greater magnification of the black square (hematoxylin-eosin, original magnification $\times 100$ ) reveals slight invasion into the tarsus (c). T, tumor; TA, tarsus.
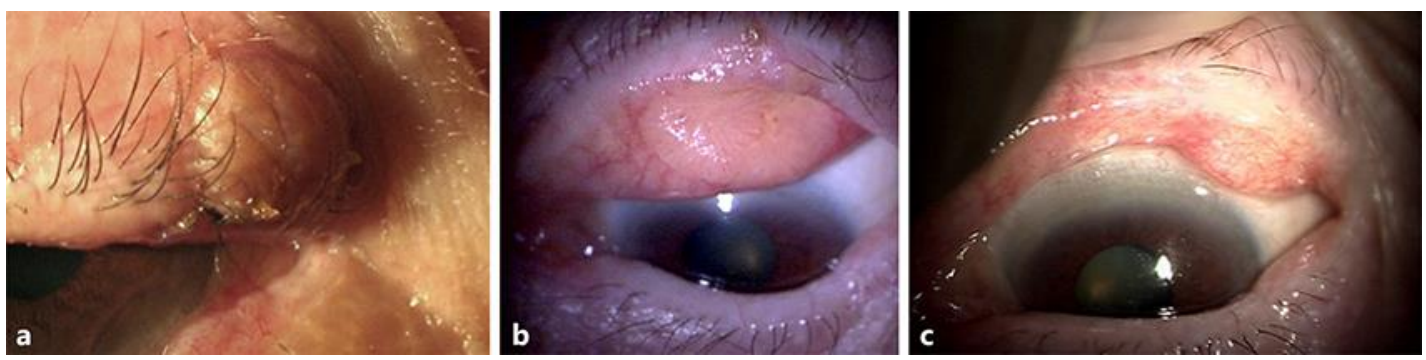

Fig. 3. The postoperative appearance of the left upper eyelid 2 weeks after the separation procedure for the switched flap from the lower eyelid (a). A Bowenoid proliferation of the recurrent sebaceous carcinoma in the middle third of the tarsal palpebral conjunctiva (b). The postoperative appearance of the tarsal palpebral conjunctiva 3 months after the resection of the re-recurrent tumor combined with mitomycin $\mathrm{C}$ and cryotherapy as well as simple resection (c). 